\title{
The Effect of Tween80 on Signal Intensity of Intact Proteins by MALDI Time-of-Flight Mass Spectrometry
}

\author{
Craig S. Brinkworth and David J. Bourne \\ Human Protection and Performance Division, Defence, Science, and Technology Organisation, Victoria, \\ Australia
}

Buffers and detergents are notorious for suppression of analyte signal in electrospray and MALDI mass spectrometry and, invariably, analysts will take steps to remove these contaminants before MS analysis. However, we have found serendipitously that protein signal with MALDI MS is improved by about an order of magnitude on the addition of small amounts of Tween80. Four charged states of BSA could easily be seen at less than $125 \mathrm{fmol} / \mathrm{spot}$ and with mixture of three proteins (BSA, trypsinogen, and protein A) the molecular ions could be detected on as little as $12.5 \mathrm{fmol}$ of spotted material (per protein) using an automated laser firing sequence. (J Am Soc Mass Spectrom 2007, 18, 102-105) (c) 2007 American Society for Mass Spectrometry

$\mathrm{T}$ The versatility of mass spectrometry for the identification and characterization of proteins continues to grow [1, 2]. This growth has resulted in mass spectrometry being used in tandem with a variety of other experimental techniques [3-5]. However, many of these techniques utilize reagents that can adversely affect the quality of mass spectra obtained by either suppressing analyte ionization or causing the formation of numerous adducts [6]. To avoid these effects, it is usually necessary to pretreat biological samples before analysis [7], a process which can lead to significant loss of analyte. In addition, it has been reported that modification of the matrix solution can be one way to suppress these effects such that MALDI-TOF MS can be obtained in the presence of nonionic detergents [8].

Our interest was to develop a confirmatory test for the presence of low level amounts of proteins using mass spectrometry. In addition, the method had to accommodate samples containing Tween80, a nonanionic detergent used in the preparation of biological buffers that assists in the solubilization of proteins [9]. Amini and coworkers [10] reported that Tween80 produced prominent spectra of its own in the presence of sodium or potassium ions to the detriment of analyte signal.

To develop our methodology for low level protein detection, we used a model protein, bovine serum albumin (BSA), and were surprised to find that the addition of Tween80, rather than leading to signal

Published online October 13, 2006

Address reprint requests to Dr. Craig S. Brinkworth, Human Protection and Performance Division, Defence Science and Technology Organisation, 506 Lorimer Street, Fishermans Bend, Victoria 3207, Australia. E-mail: craig. brinkworth@dsto.defence.gov.au degradation, produced the opposite result. In this communication, we discuss the unexpected advantageous effect of Tween 80 on the MALDI spectrum of large proteins.

\section{Materials and Methods}

$\alpha$-Cyano-4-hydroxycinammic acid (cat. no. 70990) and sinapic acid (cat. no. 85429) were obtained from Fluka (St. Louis, MO) and were both in excess of $99.6 \%$ pure. HPLC grade water was from Merck (Darmstadt, Germany). Tween80 was from Sigma (St. Louis, MO). Bovine serum albumin, high purity, was from Pharmacia (New York, NY). Protein calibration standard II (trypsinogen, protein $\mathrm{A}$, and bovine serum albumin mix) was from Bruker Daltonik (Leipzig, Germany).

\section{Preparation of Solutions of Bovine Serum Albumin (BSA) and Protein Calibration Standard II}

All the relevant stock and working solutions for each set of comparison experiments were prepared, spotted, and the mass spectra acquired on the same day to ensure, as much as possible, that the only difference between the solutions was either the presence or absence of Tween 80 . The Tween 80 stock solutions were prepared using neat Tween 80 and HPLC water (vol/vol). Solutions of bovine serum albumin were prepared in the following concentrations: $1 \mathrm{pmol} / \mu \mathrm{L}$ and 500, 250, and $125 \mathrm{fmol} / \mu \mathrm{L}$ using water and $0.01 \%$ Tween 80 solution. Briefly, stock solutions of BSA (100 pmol/ $\mu \mathrm{L})$ were prepared using lyophilized BSA and water or $0.01 \%$ Tween 80 solution. The amount of BSA in $5 \mu \mathrm{L}$ of the 
respective stock solutions was quantified using the GE Healthcare (Piscataway, NJ) 2-D Quant Kit (cat no. 80-6483-56) by following the manufacturer's instructions. The data indicated that both solutions contained the same amount of protein at the desired concentration. The $100 \mathrm{pmol} / \mu \mathrm{L}$ stock solutions were diluted with either water or $0.01 \%$ Tween 80 solution to give the desired concentrations of BSA.

Stock solutions of BSA $(100 \mathrm{pmol} / \mu \mathrm{L})$ in varying concentrations of Tween80 (10, 1, 0.1, 0.01, and 0.001\%) were prepared by dissolving lyophilized BSA in the respective Tween 80 stock solutions. The working solutions of BSA $(125 \mathrm{fmol} / \mu \mathrm{L})$ in 10, 1, 0.1, 0.01, and $0.001 \%$ Tween 80 were prepared by diluting the BSA stock solutions with the appropriate Tween 80 solution.

Stock solutions of protein calibration standard II (12 $\mathrm{pmol} / \mu \mathrm{L})$ were prepared using the lyophilized standard with water and $0.01 \%$ Tween 80 . The stock solutions were subsequently diluted with water and $0.01 \%$ Tween 80 to give working solutions of $1 \mathrm{pmol} / \mu \mathrm{L}, 500$ $\mathrm{fmol} / \mu \mathrm{L}, 100 \mathrm{fmol} / \mu \mathrm{L}$, and $50 \mathrm{fmol} / \mu \mathrm{L}$.

\section{Preparation of MALDI Target}

A MTP 384 massive target T (part no. 26755) from Bruker Daltonik (Leipzig, Germany) was used for all the experiments described in this paper. The target was spotted as follows for all the BSA experiments: the working solutions were mixed with matrix (sat. $\alpha$-cyano-4-hydroxycinnamic acid in acetonitrile: $0.1 \%$ trifluoroacetic acid, 33:67) in a 1:1 ratio for a total of $2 \mu \mathrm{L}$ and the entire mixture applied to target and allowed to air dry. For the protein calibration standard II working solutions the following protocol for spotting was followed: the working solutions were mixed with matrix (sat. $\alpha$-cyano-4-hydroxycinnamic acid in acetonitrile: $0.1 \%$ trifluoroacetic acid, 33:67) in a 1:1 ratio for a total of $1 \mu \mathrm{L}$ and two spots of $0.5 \mu \mathrm{L}$ applied to target and allowed to air dry.

\section{Acquisition of Mass Spectral Data}

MALDI mass spectra were acquired on an Autoflex II TOF-TOF mass spectrometer from Bruker Daltonik (Leipzig, Germany). The spectra were acquired in linear mode using the following settings: laser power $43 \%$ (compared with 25 to $30 \%$ for a standard tryptic digest), laser frequency: $25 \mathrm{~Hz}$, ion source 1: $20.00 \mathrm{kV}$, ion source 2: $17.50 \mathrm{kV}$, lens: $8.50 \mathrm{kV}$, pulsed ion extraction: 350 ns, matrix suppression: deflection $(600 \mathrm{~m} / \mathrm{z}), \mathrm{m} / \mathrm{z}$ detection: 10,000 to 100000, range: medium, sample rate: $0.5 \mathrm{GS} / \mathrm{s}$, detector gain: 19.0x (turbo: on), high mass accelerator: $9.75 \mathrm{kV}$, electronic gain: enhanced and real time smooth: off. An automated laser firing sequence was used to obtain the data that involved the accumulation of 3000 shots (100 shots per position) using a spiral pattern originating in the center of the spot. The shots were summed and the resulting data were

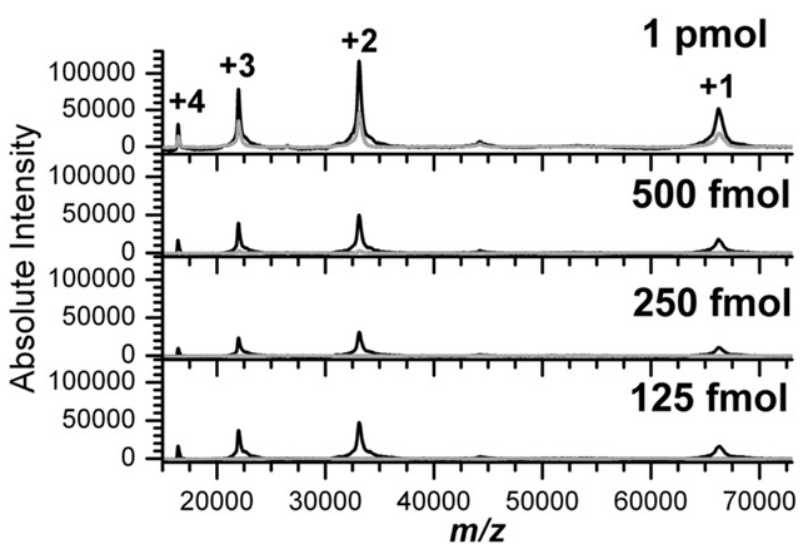

Figure 1. The MALDI mass spectra of intact bovine serum albumin (BSA) using sat. $\alpha$-cyano-4-hydroxycinnamic acid. A 1:1 ratio of analyte:matrix was used for a total of $2 \mu \mathrm{L}$. BSA was dissolved in water (grey) and $0.01 \%$ Tween80 (black). The amount of BSA spotted is indicated on the figure along with the observed charge states.

smoothed using the following parameters: algorithm: Savitzky Golay, width: $100 \mathrm{~m} / \mathrm{z}$ and cycles: 3.

\section{Results and Discussions}

The resulting mass spectra from the solutions of BSA prepared in the following concentrations: $1 \mathrm{pmol} / \mu \mathrm{L}$ and 500,250 , and $125 \mathrm{fmol} / \mu \mathrm{L}$ using both water and $0.01 \%$ Tween 80 solution are summarized in Figure 1. The grey lines represent the mass spectra acquired from BSA in water and the black lines are those acquired from BSA in $0.01 \%$ Tween 80 solution. The total amount of protein applied to the spot is indicated on each spectrum. Four charge states for BSA were observed and, surprisingly, the solutions prepared in $0.01 \%$ Tween 80 gave strong signals at $125 \mathrm{fmol}$ whereas BSA in water was not detected at this level. Importantly, the protonated molecular ion was present along with confirmatory multiply charged species, allowing for unambiguous identification of the molecular ion. Conversely, only the $1 \mathrm{pmol} \mathrm{BSA} /$ water solution gave reasonable signal of all four charge states while for the $500 \mathrm{fmol}$ only small peaks were observed for the +1 and +2 charge states. These experiments were repeated three times with the same result. The presence of Tween 80 greatly enhances the sensitivity level for intact BSA by MALDI mass spectrometry.

A clue to understanding this phenomenon was the appearance of the spots as summarized in Figure 2. The water solutions gave isolated clumps of large crystals (Figure 2a), while solutions containing $0.01 \%$ Tween 80 produced a very even mat of finer crystals (Figure 2e). To optimize the enhancement, solutions of BSA at a concentration of $125 \mathrm{fmol} / \mu \mathrm{L}$ were prepared with various percentages of Tween $80(10,1,0.1,0.01$ and $0.001 \%$ ). The targets were spotted as described in the experimental section and the results are summarized in Figure 3 . The solutions containing 10 and 1\% Tween 80 


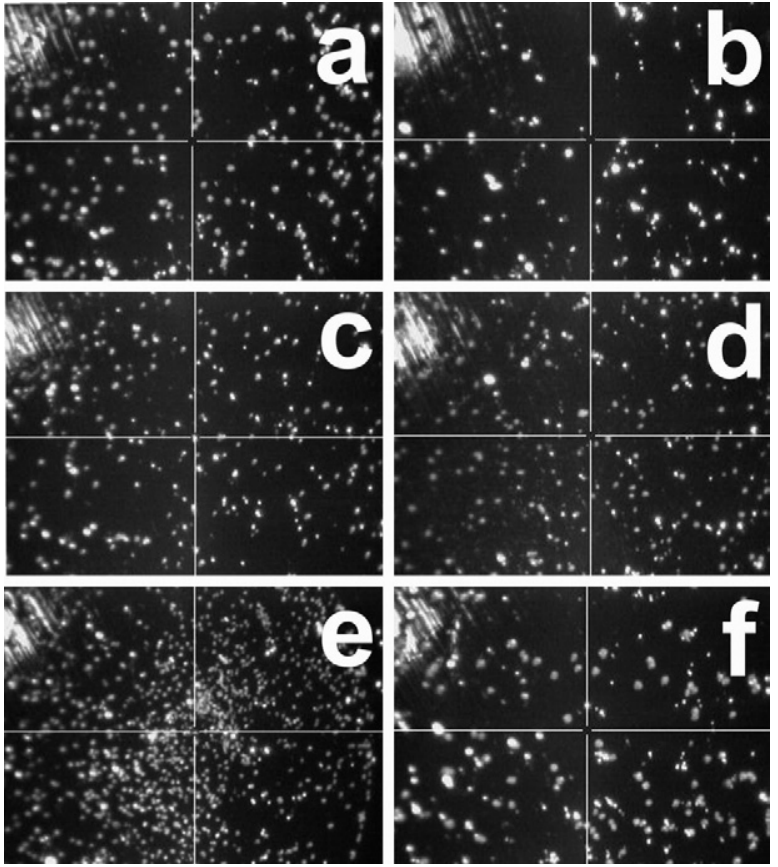

Figure 2. Images of $2 \mu \mathrm{L}$ spots as viewed by the camera on the mass spectrometer when spotted with a mixture of $1 \mu \mathrm{L}$ of sat. $\alpha$-cyano-4-hydroxycinnamic acid matrix solution (33: 67, acetonitrile: $0.1 \%$ trifluoroacetic acid) and $1 \mu \mathrm{L}$ of $125 \mathrm{fmol} / \mu \mathrm{L} \mathrm{BSA}$ in (a) water, (b) 10\% Tween 80 , (c) $1 \%$ Tween 80 , (d) $0.1 \%$ Tween 80 , (e) $0.01 \%$ Tween 80 , and (f) $0.001 \%$ Tween 80 .

gave no detectable signal. This was not totally unexpected because the spots looked quite waxy, suggesting a layer of detergent over the entire spot. This is evident from the pictures of the spots in Figure $2 b$ and $c$, respectively. In Figure $2 b$ (10\% Tween 80$)$, particularly, there are very few crystals on the spot and those that are present are large. This is true to a lesser extent for the spot using 1\% Tween 80 (Figure 2c). The spectrum of the $0.001 \%$ Tween 80 solution gave no observable signal and the spots took on the appearance of those prepared in water samples as indicated by the similarities between Figure 2a and f. Signal was observed for both the 0.1 and $0.01 \%$ solutions with the $0.01 \%$ solution giving the slightly better sensitivity. The pictures of these spots are shown in Figure $2 \mathrm{~d}$ and e, respectively. It is at this concentration that the crystals are at their finest and most evenly distributed throughout the spot.

Sinapic acid was also used as the matrix with similar results (data not shown) i.e., solutions with $0.01 \%$ Tween 80 allowed much greater sensitivity than those without. However, sinapic acid results in fewer observable charge states and with the +1 charge state being the most intense. Also, the sensitivity was about one order of magnitude less than that of $\alpha$-cyano-4-hydroxycinnamic acid. These results suggest that the effect of Tween 80 is not restricted to one particular matrix.

We believe that Tween 80 is influencing the drying of the spots of the MALDI target, resulting in the observed increase in sensitivity. First, the reduction in surface tension of the droplet results in a more even distribution of the solvent on the target. This produces a more uniform thin layer of matrix and analyte on the target upon drying. In addition, the presence of Tween 80 enables the proteins to remain in solution longer so that the protein is distributed as a fine layer rather than being deposited in spatially isolated sweet spots on the target as the drop evaporates. When $0.5 \mu \mathrm{L}$ drops are spotted, 50 laser shots will consume the protein/matrix entirely, indicating just how fine the resultant layer is. The fine homogeneous layering will inevitably give rise to greater reproducibility, even when very small amounts (low fmol) are used. This is very important for automated acquisition sequences that are capable of automatic laser tracking and firing.

Finally, we present our experiments into the efficiency of this process involving a more complex mixture of proteins at lower sample amounts. This is more akin to what may be expected from an unknown sample. Solutions containing trypsinogen (Tryp), protein A (Prot A), and BSA in equal concentrations $(1 \mathrm{pmol} / \mu \mathrm{L}$ and 500,100 , and $50 \mathrm{fmol} / \mu \mathrm{L})$ were prepared in $0.01 \%$ Tween 80 and spotted with equal amounts of matrix $(0.25 \mu \mathrm{L}$ of each) for a total volume of $0.5 \mu \mathrm{L}$. For comparison, solutions of the three proteins were also prepared in water and spotted. The resulting spectra from these spots are summarized in Figure 4 . The top two spectra represent $250 \mathrm{fmol}$ of each protein loaded in water (grey) and $0.01 \%$ Tween 80 (black). Each of the three proteins is clearly identifiable but the sensitivity of the one prepared with Tween 80 is greater. The other three spectra represent lower amounts of loaded protein prepared in $0.01 \%$ Tween 80 solution. The corresponding solutions prepared in water did not give any observable signals and are not shown. These results show that when only $12.5 \mathrm{fmol}$ of each protein is loaded on the target, it is still possible to identify the +1 charge states of each protein. In fact, the sensitivity of the $12.5 \mathrm{fmol}$ mass spectrum of the three

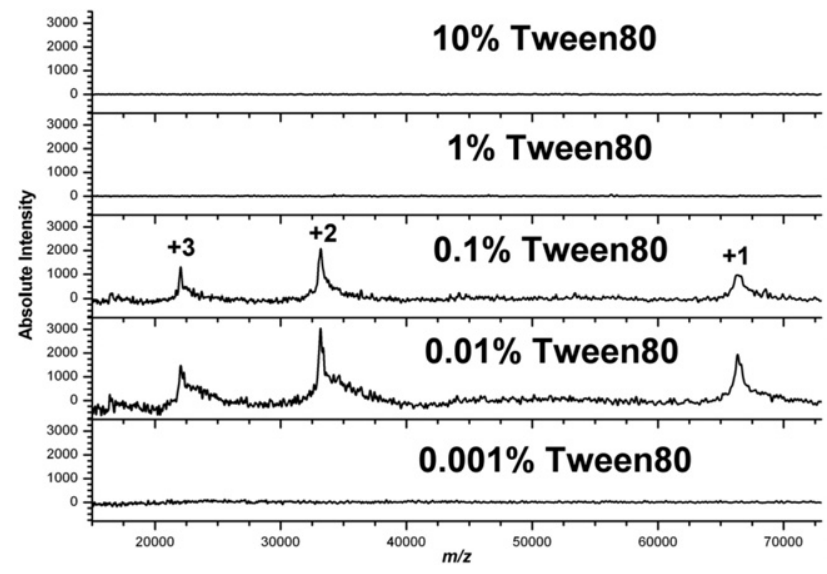

Figure 3. The MALDI mass spectra of intact bovine serum albumin (BSA) using sat. $\alpha$-cyano-4-hydroxycinnamic acid. A 1:1 ratio of analyte:matrix was used for a total of $2 \mu \mathrm{L}$. BSA (125 $\mathrm{fmol} / \mu \mathrm{L}$ ) was prepared in varying concentrations of Tween 80 . The concentration of Tween 80 is indicated on the figure along with the observed charge states. 


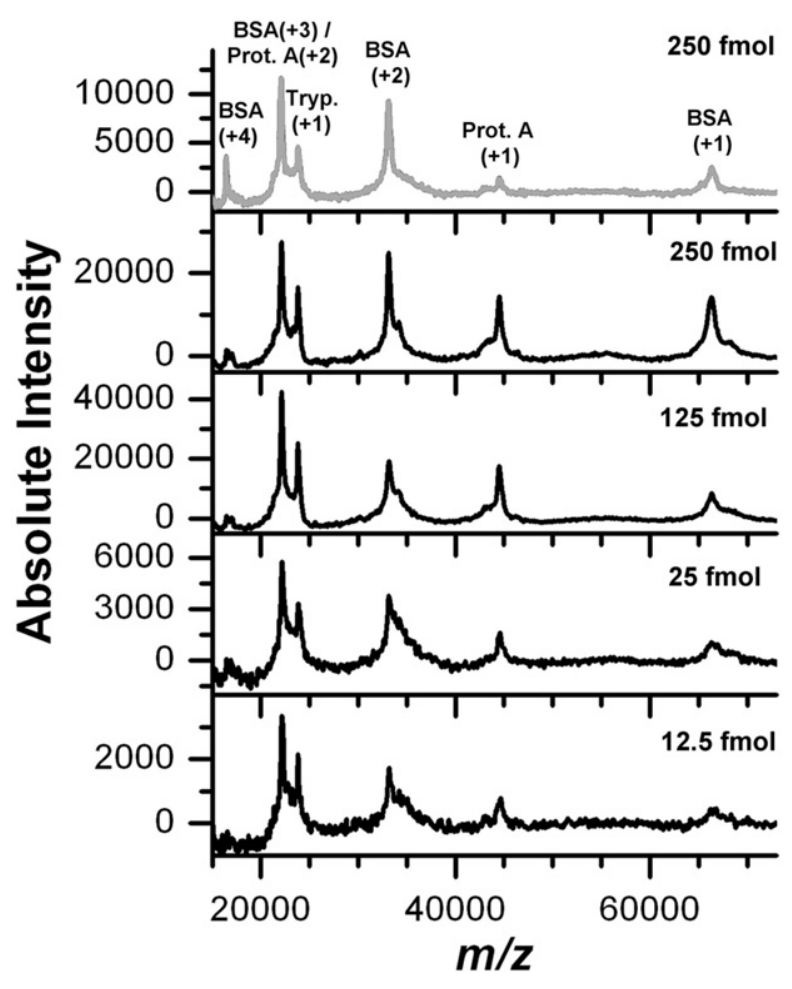

Figure 4. The intact protein MALDI mass spectra of a three protein mix, bovine serum albumin (BSA), protein A (Prot A), and trypsinogen (Tryp) of varying amounts (indicated on the figure). The solution using water is shown in grey while those using $0.01 \%$ Tween 80 are shown in black.

proteins is only a fifth of that of the 250 fmol mass spectrum prepared in water with 20 times less sample.

\section{Conclusions}

The sensitivity of intact protein MALDI mass spectrometry can be greatly enhanced by adding $0.01 \%$
Tween 80 to the analyte solution before spotting. The mass spectra are unaffected by the Tween 80 at the mass range of interest and no adverse effects are observed as have been reported for lower mass range studies [10].

\section{References}

1. Kussmann, M.; Affolter, M.; Fay, L. B. Proteomics in Nutrition and Health. Comb. Chem. High Throughput Screen 2005, 8, 679-696.

2. Chalkley, R. J.; Hansen, K. C.; Baldwin, M. A. Bioinformatic Methods to Exploit Mass Spectrometric Data for Proteomic Applications. Methods Enzymol. 2005, 402, 289-312.

3. Cargile, B. J.; Sevinsky, J. R.; Essader, A. S.; Stephenson, J. L., Jr.; Bundy, J. L. Immobilized $\mathrm{pH}$ Gradient Isoelectric Focusing as a First-Dimension Separation in Shotgun Proteomics. J. Biomol. Tech. 2005, 16, 181-189.

4. Li, J.; Martin, A.; Cox, A. D.; Moxon, E. R.; Richards, J. C.; Thibault, P. Mapping Bacterial Glycolipid Complexity Using Capillary Electrophoresis and Electrospray Mass Spectrometry. Methods Enzymol. 2005, 405, 369-397.

5. Huang, L.; Burlingame, A. L. Comprehensive Mass Spectrometric Analysis of the 20S Proteasome Complex. Methods Enzymol. 2005, 405, $187-236$.

6. Jorgensen, C. S.; Jagd, M.; Sorensen, B. K.; McGuire, J.; Barkholt, V.; Hojrup, P.; Houen, G. Efficacy and Compatibility with Mass Spectrometry of Methods for Elution of Proteins from Sodium Dodecyl SulfatePolyacrylamide Gels and Polyvinyldifluoride Membranes. Anal. Biochem. 2004, 330, 87-97.

7. Drexler, D.; Barlow, D. J.; Falk, P.; Cantone, J.; Hernandez, D.; Ranasinghe, A.; Sanders, M.; Warrack, B.; McPhee, F. Development of an On-Line Automated Sample Clean-Up Method and Liquid Chromatography-Tandem Mass Spectrometry Analysis: Application in an in Vitro Proteolytic Assay. Anal. Bioanal. Chem. 2006, 384, 1145-1154.

8. Gharahdaghi, F.; Kirchner, M.; Fernandez, J.; Mische, S. M. PeptideMass Profiles of Polyvinylidene Difluoride-Bound Proteins by MatrixAssisted Laser Desorption/Ionization Time-of-Flight Mass Spectrometry in the Presence of Nonionic Detergents. Anal. Biochem. 1996, 233, 94-99.

9. Deshpande, R. G.; Khan, M. B.; Bhat, D. A.; Navalkar, R. G. Isolation of a Contact-Dependent Hemolysin from Mycobacterium tuberculosis. J. Med. Microbiol. 1997, 46, 233-238.

10. Amini, A.; Dormady, S. J.; Riggs, L.; Regnier, F. E. The Impact of Buffers and Surfactants from Micellar Electrokinetic Chromatography on Matrix-Assisted Laser Desorption Ionization (MALDI) Mass Spectrometry of Peptides. Effect of Buffer Type and Concentration on Mass Determination by MALDI-Time-of-Flight Mass Spectrometry. J. Chromatogr. A 2000, 894, 345-355. 